\title{
Are histones real pathogenic agents in sepsis?
}

\author{
Tom van der Poll, Frank L. van de Veerdonk, Brendon P. Scicluna \\ and Mihai G. Netea
}

We thank Ginsburg and Koren for their brief overview of the potential role of extracellular histones in the pathogenesis of sepsis (Ginsburg, I. \& Koren, E. Are histones real pathogenic agents in sepsis? Nat. Rev. Immunol. http://dx.doi.org/10.1038/ nri.2017.156 (2017) $)^{1}$. Although our Review article (The immunopathology of sepsis and potential therapeutic targets. Nat. Rev. Immunol. 17, 407-420 (2017) $)^{2}$ covers a variety of immunological changes and mechanisms implicated in sepsis pathogenesis, the space allowed was not unlimited. We therefore chose to discuss damage-associated molecular patterns (DAMPs) in a general way, stating that "During sepsis, the host response is further disrupted owing to the release of ... DAMPs ... . DAMPs can activate many of the PRRs [pattern recognition receptors] that also recognize PAMPs [pathogen-associated molecular patterns], giving rise to a vicious cycle that also involves sustained immune activation and organ dysfunction" and "PRRs interact with diverse PAMPs and DAMPs, and this diversity can probably explain the similarity between the inflammatory reactions induced by different pathogens and ... different types of injury, either infectious or non-infectious" (REF. 2). We cited two Reviews from major journals to support these statements ${ }^{3,4}$, and included the role of DAMPs in sepsis pathogenesis in figure 1 . Since we fully agree that reporting should be unbiased, we would like to point out that besides histones, many other DAMPs have been implicated in sepsis pathogenesis. To name a few: high-mobility group box 1 (REF. 5), S100A8-S100A9 (REFS 6,7), cold-inducible RNA binding proteins ${ }^{8}$, mitochondrial $\mathrm{DNA}^{9}$, heat shock proteins, IL-1 $\alpha$ and IL-33 (REFS 10,11). Other important topics unfortunately could also not be discussed in our Review, including mitochondrial dysfunction ${ }^{12,13}$, oxidative stress ${ }^{12,13}$, resolution of inflammation (with the role of bioactive lipids such as lipoxins and resolvins therein $)^{14}$ and the mechanisms that could contribute to late sequelae of sepsis ${ }^{15}$. The pathogenesis of sepsis is extremely complex and variable, depending on the pathogen (load and virulence), the host (genetics, epigenetics and comorbidity), the environment (including the microbiome) and the time elapsed after the start of the infection, with distinct host responses at local, regional and systemic levels. In this respect, as we tried to accentuate in our Review, it remains unclear which mechanisms are the main drivers of sepsis-associated pathology in time.

Tom van der Poll is at the Center of Experimental \& Molecular Medicine, and the Division of Infectious Diseases, Academic Medical Center, University of Amsterdam, Meibergdreef 9, 1105AZ Amsterdam, Netherlands.

Frank $L$. van de Veerdonk is at the Department of Internal Medicine, and the Center for Infectious Diseases, Radboud University Medical Center, Geert Grooteplein Zuid 8, 6525 GA Nijmegen, Netherlands.

Brendon P. Scicluna is at the Department of Clinical Epidemiology, Biostatistics and Bioinformatics, and the Center of Experimental \& Molecular Medicine, Academic Medical Center, University of Amsterdam, Meibergdreef 9, 1105AZ Amsterdam, Netherlands.

Mihai $G$. Netea is at the Department of Internal Medicine, and the Center for Infectious Diseases, Radboud University Medical Center, Geert Grooteplein Zuid 8, 6525 GA Nijmegen, Netherlands.

Correspondence to T.v.d.P. t.vanderpoll@amc.uva.n! doi: $10.1038 /$ nri.2017.157 Published online 27 Dec 2017
1. Ginsburg, I. \& Koren, E. Are histones real pathogenic agents in sepsis? Nat. Rev. Immunol. http://dx.doi. org/10.1038/nri.2017.156 (2017)

2. van der Poll, T. et al. The immunopathology of sepsis and potential therapeutic targets. Nat. Rev. Immunol. 17, 407-420 (2017).

2. Chan, J. K. et al. Alarmins: awaiting a clinical response. J. Clin. Invest. 122, 2711-2719 (2012).

3. Deutschman, C. S. \& Tracey, K. J. Sepsis: current dogma and new perspectives. Immunity 40, 463-475 (2014).

4. Andersson, U. \& Tracey, K. J. HMGB1 is a therapeutic target for sterile inflammation and infection. Annu. Rev. Immunol. 29, 139-162 (2011).

5. Vogl, T. et al. Mrp8 and Mrp14 are endogenous activators of Toll-like receptor 4, promoting lethal, endotoxin-induced shock. Nat. Med. 13, 1042-1049 (2007).

6. van Zoelen, M. A. et al. Expression and role of myeloid-related protein-14 in clinical and experimental sepsis. Am. J. Respir. Crit. Care Med. 180, 1098-1106 (2009).

7. Oiang, X. et al. Cold-inducible RNA-binding protein (CIRP) triggers inflammatory responses in hemorrhagic shock and sepsis. Nat. Med. 19, 1489-1495 (2013).

8. Boyapati, R. K. et al. Advances in the understanding of mitochondrial DNA as a pathogenic factor in inflammatory diseases. F1000Res 6, 169 (2017)

9. Xu, H. et al. Role of the IL-33-ST2 axis in sepsis. Mil. Med. Res. 4, 3 (2017).

10. Kang, J. W. et al. DAMPs activating innate immune responses in sepsis. Ageing Res. Rev. 24, 54-65 (2015).

11. Singer, $\mathbf{M}$. The role of mitochondrial dysfunction in sepsis-induced multi-organ failure. Virulence 5, 66-72 (2014).

12. Arulkumaran, N. et al. Mitochondrial function in sepsis. Shock 45, 271-281 (2016).

13. Buechler, C., Pohl, R. \& Aslanidis, C. Pro-resolving molecules - new approaches to treat sepsis? Int J. Mol. Sci. 18, E476 (2017).

14. Shankar-Hari, M. \& Rubenfeld, G. D. Understanding long-term outcomes following sepsis: implications and challenges. Curr. Infect. Dis. Rep. 18, 37 (2016).

Competing interests statement

The authors declare no competing interests. 